\title{
A Class of Analytic Perturbations for One-body Schrödinger Hamiltonians
}

\author{
J. Aguilar and J. M. Combes \\ Centre de Physique Théorique - C.N.R.S., Marseille, France
}

Received March 2, 1971

\begin{abstract}
We study a class of symmetric relatively compact perturbations satisfying analyticity conditions with respect to the dilatation group in $R^{n}$. Absence of continuous singular part for the Hamiltonians is proved together with the existence of an absolutely continuous part having spectrum $[0, \infty)$. The point spectrum consists in $\mathbb{R}-\{0\}$ of finite multiplicity isolated energy bound-states standing in a bounded domain. Bound-state wave functions are analytic with respect to the dilatation group. Some properties of resonance poles are investigated.
\end{abstract}

\section{Introduction}

The recent developments of scattering theory for long range potentials ([1-3]) have made urgent to find new techniques for the spectral analysis of Schrödinger Hamiltonians. By new we mean independent of a short range hypothesis implying e.g. existence of the usual wave operators or (almost equivalently) solvability of the Lippman-Schwinger equation. Many results have been found recently by using methods related to Putnam's positive commutator theorems (to our knowledge the most recent of them can be found in $[4,5]$ which also contains many references). We present here a different method based upon rather weak analyticity conditions on the potentials. These conditions allow analytic continuation on the unphysical sheet for sufficiently many expectation values of the resolvent; from this all qualitative results on the spectrum can be deduced. Our conditions allow velocity dependent perturbations such as spin-orbit couplings or electromagnetic fields. As a consequence we don't expect absence of positive discrete spectrum. A part of this work will be concerned with a study of positive energy bound-states and corresponding eigenfunctions. Finally, we investigate properties of poles on the unphysical sheet.

\section{Dilatation Analytic Potentials}

The dilatation group in $L^{2}\left(R^{n}\right)$ is defined as

$$
(U(\theta) \Phi)(x)=\epsilon^{n \theta / 2} \Phi\left(e^{\theta} x\right) \quad \theta \in R, \Phi \in L^{2}\left(R^{n}\right) .
$$


In momentum space $U(\theta)$ acts as follows:

$$
(U(\theta) \Phi)(p)=e^{-n \theta / 2} \Phi\left(e^{-\theta} p\right) .
$$

The proof that $U(\theta), \theta \in R$, is a weakly continuous unitary group is rather straightforward and will be omitted. The generator of this group is $A=x \cdot P+P \cdot x$ (called a "progress operator" in [4]) which also enters in the study of the virial theorem [6]). From (2) we immediately deduce that

$$
U(\theta) R_{0}(z) U(\theta)^{-1}=e^{2 \theta} R_{0}\left(e^{2 \theta} z\right)
$$

where $R_{0}(z)=\left(H_{0}-z\right)^{-1}, \operatorname{Im} z \neq 0$; and $H_{0}=-\Delta$ is the $n$-dimensional Laplacian. Relation (3) has a fundamental consequence which is the invariance under $U(\theta), \theta \in R$, of the Hilbert space $\mathscr{D}\left(H_{0}\right)$, (the domain of $H_{0}$ equipped with the scalar product $(\Phi, \Psi)_{\mathscr{D}\left(H_{0}\right)}=\left(H_{0} \Phi, H_{0} \Psi\right)+(\Phi, \Psi)$, $\left.\Phi, \Psi \in \mathscr{D}\left(H_{0}\right)\right)$. More precisely one has:

$$
\|U(\theta)\|_{\mathscr{D}\left(H_{0}\right)}=e^{2 \theta}, \theta \in R .
$$

Let us now consider an $H_{0}$-compact operator $V$ (that is a compact operator from $\mathscr{D}\left(H_{0}\right)$ to $\left.L^{2}\left(R^{n}\right)\right)$ and define

$$
V(\theta)=U(\theta) V U(\theta)^{-1}, \quad \theta \in R .
$$

It is clear that $V(\theta)$ is $H_{0}$-compact. We are then sufficiently prepared to define dilatation analytic perturbations as those $H_{0}$-compact operators $V$ having the following property:

$V(\theta), \theta \in R$, has an $H_{0}$-compact analytic continuation in an open connected domain $\mathcal{O}$ of the complex plane.

If $V(\theta), \theta \in R$, is symmetric the domain $\mathcal{O}$ can always be taken to be symmetric with respect to $R$ since

$$
V^{*}(\bar{\theta})=V(\theta), \quad \theta \in \mathcal{O}
$$

defines an analytic continuation of $V(\theta)$ into the symmetric domain generated by $\mathcal{O}$. Furthermore we see that

$$
V_{\theta_{0}}(\theta)=U\left(\theta_{0}\right) V(\theta) U^{-1}\left(\theta_{0}\right) \quad \theta \in \mathcal{O}, \theta_{0} \in R
$$

defines for a fixed $\theta_{0}$ another family of $H_{0}$-compact operators analytic in $\mathcal{O}+\theta_{0}$. It is clear that we can in this way extend analytically $V(\theta)$ to the whole strip generated by translating $\mathcal{O}$ along the real axis; in fact it is sufficient to verify that $V(\theta)$ and $V_{\theta_{0}}(\theta)$ coincide on the real intersection of $\mathcal{O}$ and $\mathcal{O}+\theta_{0}$ for sufficiently small $\theta_{0}$. Summing up we have:

Lemma I.1. Assume $V$ is symmetric and dilatation analytic; the analyticity domain $\mathcal{O}$ for $V(\theta)$ can always be extended to a complex strip

$$
S_{a}=\{z \in \mathbb{C},-a<\operatorname{Im} z<+a\}, \quad a>0 .
$$


In the following we shall always assume $0<a<\Pi / 4$.

Some standard examples of dilatation analytic perturbations are $g / r^{\beta}(0<\beta<3 / 2)$ or $p \rightarrow p-e A$ where $A=g / r^{\beta}(0<\beta<3 / 4)$. There also exists short range potentials satisfying this kind of analyticity such as superpositions of Yukawa potentials studied by Lovelace [7]. In fact the idea of using complex scale transformations in perturbation theory is not new and as far as we know this been originated in papers [8] and [9] in order to study analyticity properties of scattering amplitudes. Lovelace has also shown the interest of such transformations for the study of resonance and three-particle amplitudes.

\section{Spectral Type of $\mathrm{H}=\mathrm{H}_{0}+\mathrm{V}$}

Let $\mathscr{D}$ be the dense set of analytic vectors for the dilatation group; $\mathscr{D}$ consists of those vectors $\Phi$ such that the series $\sum_{n} \frac{\left\|A^{n} \Phi\right\|}{n !} \theta^{n}$ has an infinite radius of convergence [12]. We shall denote by $\Phi(\theta), \theta \in \mathbb{C}$ the corresponding vector valued analytic function. More generally if $\mathcal{O}$ is any open complex domain having a non empty intersection with $R$, then $\mathscr{D}(\mathcal{O})$ will consist of those vectors $\Phi$ such that $\Phi(\theta)=U(\theta) \Phi, \theta \in R$ can be analytically continued in $\mathcal{O}$.

Lemma II.1. Let $V$ be symmetric and dilatation analytic in a strip $S_{a}$. Let $R(z)=\left(H_{0}+V-z\right)^{-1}, \operatorname{Im} z \neq 0$; then for any $\Phi \in \mathscr{D}$, the function $(\Phi, R(z) \Phi)$ has a meromorphic continuation through the positive real axis from above (resp. below) until the half lines $\arg z=-2 a($ resp. $+2 a)$.

Proof. We first notice that $H$ is self-adjoint and that $\mathscr{D}(H)=\mathscr{D}\left(H_{0}\right)$. Let us define the following subsets of the complex plane $\mathbb{C}$ :

$$
\begin{aligned}
\mathbb{C}_{[0, \infty)} & =\{z \in \mathbb{C}, z \notin[0, \infty)\}, \\
\mathbb{C}^{++} & =\{z \in \mathbb{C}, \operatorname{Re} z>0, \operatorname{Im} z>0\}, \\
\mathbb{C}^{+-} & =\{z \in \mathbb{C}, \operatorname{Re} z>0, \operatorname{Im} z<0\} .
\end{aligned}
$$

We have according to the $H_{0}$-compactness of $V$ :

$$
(\Phi, R(z) \Phi)=\left(\Phi, R_{0}(z)\left[1+V R_{0}(z)\right]^{-1} \Phi\right) \forall \Phi \in L^{2}\left(R^{n}\right), \quad z \in \mathbb{C}_{[0, \infty)} .
$$

In fact $V R_{0}(z)$ is a compact operator valued analytic function in $\mathbb{C}_{[0, \infty)}$ whose norm is smaller than one for $\operatorname{Re} z$ sufficiently large and negative. Then, as is proved for example in ([11], VII.6.12) $\left[1+V R_{0}(z)\right]^{-1}$ exists and is meromorphic in the analyticity domain of $V R_{0}(z)$. On the other hand since $\mathscr{D}\left(H_{0}\right)$ is invariant under $U(\theta), \theta \in R$, one has:

$$
U(\theta) V R_{0}(z) U(\theta)^{-1}=e^{2 \theta} V(\theta) R_{0}\left(e^{2 \theta} z\right) .
$$


Accordingly for any $\theta \in R$ :

$$
(\Phi, R(z) \Phi)=e^{2 \theta}\left(\Phi(\theta), R_{0}\left(e^{2 \theta} z\right)\left[1+e^{2 \theta} V(\theta) R_{0}\left(e^{2 \theta} z\right)\right]^{-1} \Phi(\theta)\right) .
$$

Let us suppose now that $\Phi \in \mathscr{D}$ and let $\mathcal{O}$ be the analyticity domain for $V(\theta)$. We define:

$$
\mathcal{O}^{\varepsilon}=\{z \in \mathcal{O}, \operatorname{Im} z>\varepsilon\} .
$$

We shall always assume without restricting the generality that $\mathcal{O}$ is contained in a strip $[-i a,+i a]$ with $a<\Pi / 4$. Now fix $z \in \mathbb{C}^{++}$and define $\varepsilon=-\frac{1}{2} \arg z$; then $e^{2 \theta} z \in \mathbb{C}_{[0, \infty)}$ for any $\theta \in \mathcal{O}^{\varepsilon}$. Accordingly $e^{2 \theta} R_{0}\left(e^{2 \theta} z\right)$ and $e^{2 \theta} V(\theta) R_{0}\left(e^{2 \theta} z\right)$ are analytic in $\mathcal{O}^{\theta}$. We want to derive meromorphy of $\left[1+e^{2 \theta} V(\theta) R_{0}\left(e^{2 \theta} z\right)\right]^{-1}$ in $\mathcal{O}^{\varepsilon}$. According to the compactness of $e^{2 \theta} V(\theta) R_{0}\left(e^{2 \theta} z\right)$ this will hold if this inverse exists for some $\theta \in \mathcal{O}^{\varepsilon}[10]$; but this is true for $\theta=0$ since otherwise $H_{0}+V$ would have a complex eigenvalue $z$. From this we can deduce that if $\Phi \in \mathscr{D}$ the function

$$
\Psi_{z}(\theta)=\left(\Phi(\theta), e^{2 \theta} R_{0}\left(e^{2 \theta} z\right)\left[1+e^{2 \theta} V(\theta) R_{0}\left(e^{2 \theta} z\right)\right]^{-1} \Phi(\theta)\right)
$$

is meromorphic in $\theta \in \mathcal{O}^{z}$. Furthermore according to (6) $\Psi_{z}(\theta)$ is constant for $\theta \in \mathcal{O}^{\varepsilon} \cap R$; then it is constant for any $\theta \in \mathcal{O}^{\varepsilon}$ and in particular we have:

$$
\Psi_{z}(\theta)=(\Phi, R(z) \Phi) \quad \forall z \in \mathbb{C}^{++}, \forall \theta \in \mathcal{O}^{0} .
$$

We now fix $\theta \in \mathcal{O}^{+}=\{\theta \in \mathcal{O}, \operatorname{Im} \theta>0\}$. Then $\Psi_{z}(\theta)$ is meromorphic as a function of $z$ in

$$
\mathbb{C}_{\theta}^{++}=\left\{z \in \mathbb{C} \mid e^{2 \theta} z \in \mathbb{C}^{++}\right\}
$$

for $R_{0}\left(e^{2 \theta} z\right)$ and $V(\theta) R_{0}\left(e^{2 \theta} z\right)$ are analytic in this domain. Since $\mathbb{C}^{++} \cap \mathbb{C}_{\theta}^{++}$is open and non empty, $\Psi_{z}(\theta)$ defines an analytic continuation of $(\Phi, R(z) \Phi)$ in this domain. On the other hand $\mathbb{C}_{\theta}^{++}$contains the positive real axis; we can conclude that $(\Phi, R(z) \Phi)$ can be analytically continued when $z$ crosses the positive real axis from above. Taking $\theta=+i a$ provides an analytic continuation of $(\Phi, R(z) \Phi)$ in $\mathbb{C}_{i a}^{++}$. One proves similarly the existence of an analytic continuation for $(\Phi, R(z) \Phi)$ when $z$ crosses the real axis from below.

The discussion of Lemma II.1 shows that for $\theta \in \mathcal{O}$ positive real poles of the meromorphic family $\left[1+e^{2 \theta} V(\theta) R_{0}\left(e^{2 \theta} z\right)\right]^{-1}, \quad z \in \mathbb{C}_{\theta}^{++}$, can accumulate at most at point zero. Furthermore as shown in App. I, these poles do not depend on $\theta \in \mathcal{O}^{+}$. Let us then define

$$
\Sigma^{+}=\left\{\text {real poles of }\left[1+e^{2 \theta} V(\theta) R_{0}\left(e^{2 \theta} z\right)\right]^{-1}, \theta \in \mathcal{O}^{+}, z \in \mathbb{C}_{\theta}^{++}\right\} .
$$

Obviously $\Sigma^{+}$coincides with the set of real poles of the family of resolvents $\left(e^{2 \theta} H_{0}+V(\theta)-z\right)^{-1}$. Such poles are simple. This can be proved along the lines developped in the next lemma of this paper. 
Lemma II.2. $\Sigma^{+}$is the positive point spectrum of $H$.

Proof. Let us denote by $P(E)$ the spectral family of $H$; then one has:

$$
(\Phi[P(E)-P(E-0)] \Psi)=\lim _{z \rightarrow E}(z-E)(\Phi, R(z) \Psi), \quad z \in \mathbb{C}_{\theta}^{++} .
$$

For $\theta \in \mathcal{O}^{+}$and $E \in \Sigma^{+}$we define projection operator

$$
P^{+}(E, \theta)=\underset{z=E}{\operatorname{Res}}\left(e^{-2 \theta} H_{0}+V(\theta)-z\right)^{-1} \text {. }
$$

Properties of these operators are investigated in App. I. According to (7) and (8) one gets since $E$ is a simple pole of $(\Phi, R(z) \Psi)$ :

$$
\lim _{z \rightarrow E}(z-E)(\Phi, R(z) \Psi)=\left(\Phi(\bar{\theta}), P^{+}\{E, \theta\} \Psi(\theta)\right), \quad \Phi, \Psi \in \mathscr{D} .
$$

The right member of (12) cannot vanish for all $\Phi, \Psi \in \mathscr{D}$ unless $P^{+}(E, \theta)=0$ which would contradict $E \in \Sigma^{+}$. According to (10) for such vectors $(\Phi,[P(E)-P(E-0)] \Psi)$ is non zero, which means that $E$ is in the point spectrum of $H$. Conversely assume that $E$ is not in $\Sigma^{+}$; then since $(\Phi,[P(E)-P(E-i 0)] \Psi)$ vanishes for any $\Phi, \Psi$ in $\mathscr{D}$, one has $P(E)$ $=P(E-i 0)$ which implies that $E$ is not in the point spectrum of $H$.

\section{Lemma II.3. The point spectrum of $H$ is bounded.}

Proof. Since $V(\theta)$ is $H_{0}$-compact for any $\theta \in S_{a}$ one has the following classical estimates

$$
\|V(\theta) f\| \leqq \varepsilon(\theta)\left\|H_{0} f\right\|+b(\varepsilon, \theta)\|f\|, \quad f \in \mathscr{D}\left(H_{0}\right)
$$

where $\varepsilon(\theta)$ can be choosen arbitrarily small [13]. Taking $\theta=0$, such an estimate is sufficient to prove boundedness of the negative discrete spectrum of $H$; since this result is well-known we shall investigate only the positive discrete spectrum. It is clear that majorizing $\left\|e^{2 \theta} V(\theta) R_{0}\left(e^{2 \theta} E\right)\right\|$ by 1 for sufficiently large $E \in R$ and fixed $\theta \in \mathcal{O}^{+}$is sufficient to conclude. Taking $\theta=i a$ (we assume $0<a<\Pi / 4$ ) leads after some simple calculations that we omit to

$$
\left\|V(i a) R_{0}\left(e^{2 i a} E\right)\right\| \leqq \frac{1}{\sin 2 a}\left[\varepsilon(i a)+\frac{b(\varepsilon, a)}{E}\right] .
$$

Since $\varepsilon(i a)$ can be chosen arbitrarily small the proof is complete.

We can now state the first theorem on the spectral type of the Hamiltonians constructed with dilatation analytic potentials. It is well-known that $L^{2}\left(R^{n}\right)$ can be written as a direct sum $([14], \mathrm{X}, \S 1)$

$$
L^{2}\left(R^{n}\right)=\mathscr{M}_{a}(H) \oplus \mathscr{M}_{c s}(H) \oplus \mathscr{M}_{d}(H)
$$

where $\mathscr{M}_{a}(H)\left(\operatorname{resp} . \mathscr{M}_{c s}(H), \mathscr{M}_{d}(H)\right)$ is the closed subspace of vectors $\Phi$ such that the measures $(\Phi, P(E) \Phi)$ are absolutely continuous with respect to the Lebesgue measure (resp. continuous singular, discrete). 
Theorem II.1. Let $V$ be symmetric and dilatation analytic. Then

$$
L^{2}\left(R^{n}\right)=\mathscr{M}_{a}(H) \oplus \mathscr{M}_{d}(H)
$$

the point spectrum of $H$ in $\mathbb{R}-\{0\}$ is bounded and consists of isolated eigenvalues having zero as only possible accumulation point. The restriction of $H$ to $\mathscr{M}_{a}(H)$ has spectrum $[0, \infty)$.

Proof. If $\Phi \in \mathscr{M}_{a}(H)$ then the Radon-Nikodym derivative of $(\Phi, P(\Delta) \Phi)$ with respect to Lebesgue measure is given by

$$
2 \Pi \frac{d}{d \lambda}(\Phi, P(\lambda) \Phi)=\lim _{\varepsilon \downarrow 0}(\Phi,[R(\lambda+i \varepsilon)-R(\lambda-i \varepsilon)] \Psi) .
$$

For a general $\Phi \in L^{2}\left(R^{n}\right)$, the right member of (15) defines almost everywhere the Radon-Nikodym derivative of the absolutely continuous part of the measure $(\Phi, P(\Delta) \Phi)$. The set of points where this limit does not exist is a support of the singular part of $(\Phi, P(\Delta) \Phi)$. We now choose $\Phi$ in the dense set $\mathscr{D}$; then according to Lemma II.2, the positive support of the singular part of $(\Phi, P(\Delta))$ consists of $\Sigma^{+}$; as to negative support it consists of the isolated eigenvalues of $H$ since the essential spectrum is invariant under a relatively compact perturbation. As a consequence $(\Phi, P(\Delta) \Phi)$ has no continuous singular part and accordingly $\mathscr{D} \subset \mathscr{M}_{a}(H) \oplus \mathscr{M}_{d}(H)$. Since $\mathscr{D}$ is dense and $\mathscr{M}_{a}(H) \oplus \mathscr{M}_{d}(H)$ is closed, we obtain the first assertion of the theorem. Accordingly the essential spectrum of $H$ which is $[0, \infty)$ is the union of the spectrum of $H$ restricted to its absolutely continuous part $\mathscr{M}_{a}(H)$ and of the limit points (or points with infinite multiplicities ${ }^{1}$ ) of the spectrum of $H$ restricted to $\mathscr{M}_{d}(H)$; but according to Lemma II.2, this last category contains only zero. Accordingly the last part of the theorem is verified.

\section{Properties of Bound-States}

Theorem III.1. Eigenspaces of $H$ associated to nonzero eigenvalues are finite dimensional. Bound-states wave-functions are in $\mathscr{D}(\mathcal{O})$.

Proof. Let $E$ be in the point spectrum of $H$ and let us consider the corresponding analytic families of projection operators $P^{ \pm}\{E, \theta\}, \theta \in \mathcal{O}^{ \pm}$ (App. I). If $P\{E\}$ is the projection operator (hermitian) on the eigenspace of $H$ corresponding to eigenvalue $E$, we can rewrite (12) as:

$$
\left.\left(\Phi, P^{ \pm}\{E, \theta\} \Psi\right)=(\Phi(\overline{-\theta}), P\{E\} \Psi(-\theta)\}\right) ; \quad \Phi, \Psi \in \mathscr{D} ; \theta \in \mathcal{O}^{+} .
$$

If we define now

$$
P\left\{E, \theta_{0}\right\}=U\left(\theta_{0}\right) P\{E\} U\left(\theta_{0}\right\}^{-1}, \quad \theta_{0} \in \mathcal{O} \cap R
$$

${ }^{1}$ We shall see later that this last class is empty. 
then (16) implies owing to analyticity of $\Phi$ and $\Psi$ :

$$
\lim _{\substack{\theta \rightarrow \theta_{0} \\ \theta \in \mathcal{O}^{ \pm}}}\left(\Phi, P^{ \pm}\{E, \theta\} \Psi\right)=\left(\Phi, P\left\{E, \theta_{0}\right\} \Psi\right) ; \quad \Phi, \Psi \in \mathscr{D} .
$$

Accordingly (16) defines a function $f_{\Phi \Psi}(\theta)$ analytic in $\mathcal{O}$. Our aim is to show that similarly the families $P^{ \pm}\{E, \theta\}$ and $P\{E, \theta\}$ are analytic continuation of each other. First of all, let us show boundedness of $f_{\Phi \Psi}$ in $\mathcal{O}$; one has according to (16):

$$
\left|f_{\Phi \Psi}(\theta)\right| \leqq\|\Phi(-\bar{\theta})\|\|\Psi(-\theta)\|, \quad \theta \in \mathcal{O} .
$$

If $S_{a}$ denotes the symmetric strip generated by $\mathcal{O}$ then as is shown in App. II the above inequality implies:

$$
\left|f_{\Phi \Psi}(\theta)\right|^{2} \leqq\left(\|\Phi(-i a)\|^{2}+\|\Phi(i a)\|^{2}\right)\left(\|\Psi(-i a)\|^{2}+\|\Psi(+i a)\|^{2}\right) .
$$

We need now to assume that $\mathcal{O}$ is replaced by the complex strip $S_{a}$ according to Lemma I.1. Using boundedness of $f_{\Phi \Psi}(\theta)$ in $S_{a}$, we can write integral representations for its derivatives on the real axis as follows:

$$
\left(\frac{d}{d \theta}\right)^{n} f_{\Phi \Psi}\left(\theta_{0}\right)=\frac{n !}{2 i \Pi} \int_{\Gamma_{\varepsilon}} \frac{f_{\Phi \Psi}(\theta)}{\left(\theta-\theta_{0}\right)^{n+1}} d \theta
$$

where $\Gamma_{\varepsilon}$ is the infinite contour obtained in the limit $R=\infty$ of the contour shown in the figure and $\varepsilon$ can be choosen arbitrarily close to $a$.

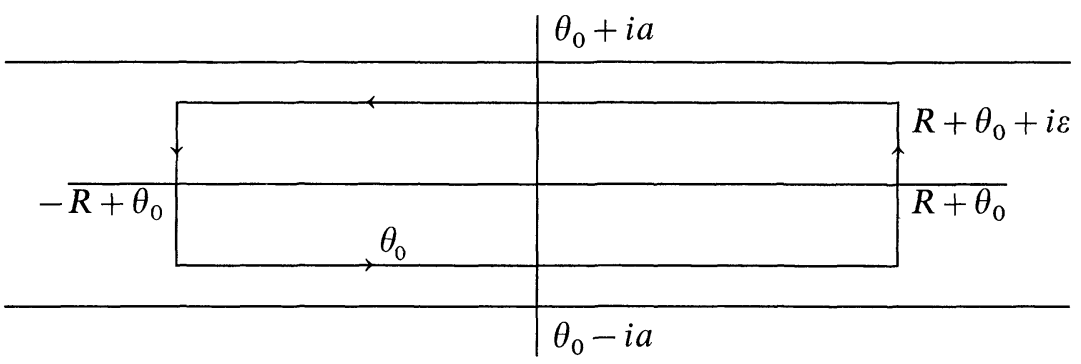

We now use the boundedness of $f_{\Phi \Psi}(\theta)$ on the vertical parts of the contour and the boundedness of $\left\|P^{ \pm}(E, \theta)\right\|$ on the horizontal parts of the contour to derive the following uniform estimates from (16):

$$
\left|\left(\frac{d}{d \theta}\right)^{n} f_{\Phi \Psi}\left(\theta_{0}\right)\right| \leqq M a^{-n} n !\|\Phi\|\|\Psi\|
$$

where $M$ is independent of $\Phi, \Psi$ and $n$. Accordingly $\left(\frac{d}{d \theta}\right)^{n}\left(\Phi, P\left\{E, \theta_{0}\right\} \Psi\right)$ can be extended to a bounded sesquilinear functional on $L^{2}\left(R^{n}\right)$ defining a bounded operator which we denote by $P^{(n)}\left\{E, \theta_{0}\right\}$

$$
\| P^{(n)}\left\{E, \theta_{0} \| \leqq M a^{-n} n ! .\right.
$$


Let

$$
Q\{E, \theta\}=\sum_{n=0}^{\infty} \frac{\left(\theta-\theta_{0}\right)^{n}}{n !} P^{(n)}\left\{E, \theta_{0}\right\}, \quad\left|\theta-\theta_{0}\right|<a .
$$

Then $Q\{E, \theta\}$ defines a bounded analytic family of operators in the domain $\left|\theta-\theta_{0}\right|<a$. Furthermore let $\boldsymbol{D}_{\theta_{0}, a}^{ \pm}=\left\{\theta \in \mathcal{O}^{ \pm},\left|\theta-\theta_{0}\right|<a\right\}$. Then the equality

$$
(\Phi, Q\{E, \theta\} \Psi)=\left(\Phi, P^{ \pm}\{E, \theta\} \Psi\right) \quad \forall \Phi, \Psi \in \mathscr{D}, \theta \in \boldsymbol{D}_{\theta_{0}, a}^{ \pm}
$$

immediately implies equality of operators. A first consequence of this equality is the norm convergence

$$
\lim _{\substack{\theta \rightarrow \theta_{0} \\ \theta \in \boldsymbol{D}_{0, a}^{ \pm}}}\left\|P\left\{E, \theta_{0}\right\}-P^{ \pm}\{E, \theta\}\right\|=0
$$

according to which $P\left\{E, \theta_{0}\right\}, \theta_{0} \in R$, (and consequently $P\{E\}(18)$ ) is compact as a uniform limit of compact operators. The second consequence is the fact that $P\left\{E, \theta_{0}\right\}, \theta_{0} \in R$, has an analytic continuation in $\mathcal{O}$ which equals $P^{+}\{E, \theta\}$ (resp. $P^{-}\{E, \theta\}$ ) in $\mathcal{O}^{+}$(resp. $\mathcal{O}^{-}$). By standard arguments (see e.g. [14], II, 6) bound-state wave-functions share analyticity properties of $P\{E, \theta\}$ that is they are in $\mathscr{D}(\mathcal{O})$.

\section{Properties of Poles on the Unphysical Sheet}

Let us make some observations about complex poles $E=M-i \Gamma$ of the meromorphic family $\left[1+e^{2 \theta} V(\theta) R_{0}\left(e^{2 \theta} z\right)\right]^{-1}, 2 \operatorname{Im} \theta>|\arg E|$. Following Lovelace we identify such poles as resonances; in fact for short-range potentials they' give rise to singularities of the scattering amplitude. Two important points must be noticed. First such poles appear only in a bounded domain of the complex plane as can be seen by a trivial extension of Lemma II.3. Secondly their degeneracy is finite.

In order to demonstrate this last property we first remark that arguments similar to those of App. I lead immediately to the result that

$$
\left(e^{-2 \theta} H_{0}+V(\theta)-z\right)^{-1}=e^{2 \theta} R_{0}\left(e^{2 \theta} z\right)\left[1+e^{2 \theta} V(\theta) R_{0}\left(e^{2 \theta} z\right)\right]^{-1}
$$

has $\theta$ independent poles in $\mathbb{C}_{i \varepsilon}^{++}$as long as $\theta \in \mathcal{O}^{+\varepsilon}$. For the purpose of analysing a given pole $E=M-i \Gamma$ we need $\varepsilon=-\frac{1}{2} \arg E$. Let us write the Laurent expansion around $E$ :

where

$$
\left(e^{-2 \theta} H_{0}+V(\theta)-z\right)^{-1}=\sum_{n=-v(E)}^{\infty}(z-E)^{n} B_{n}(E, \theta)
$$

$$
B_{n}(E, \theta)=\frac{1}{2 i \Pi} \int_{\Gamma_{E}}(z-E)^{-n-1}\left(e^{-2 \theta} H_{0}+V(\theta)-z\right)^{-1} d z
$$


and $\Gamma_{E} \in D_{i \varepsilon}^{++}$encloses only the pole $E$. We refer to Wong for the following relations

$$
\begin{aligned}
& B_{-1}(E, \theta)=B_{-1}^{2}(E, \theta)=P\{E, \theta\}, \\
& B_{-m}(E, \theta)=[N(E, \theta)]^{m-1}
\end{aligned}
$$

where $N(E, \theta)$ is the nilpotent operator

$$
[N(E, \theta)]^{v(E)}=0 .
$$

Trivial algebraic manipulations show that $P\{E, \theta\}$ and $N(E, \theta)$ are compact operators. Noticing the analyticity of $P\{E, \theta\}$ in $\theta$ then leads to the result that the constant dimension of the range of $P\{E, \theta\}$ is finite and accordingly that the degeneracy of the resonance is finite. Now obviously $E$ is a simple pole if and only if $N(E, \theta)=0$ for some $\theta, \operatorname{Im} \theta>-\frac{1}{2} \operatorname{Arg} E$. This property would garantee a Breit-Wigner form for the resonant parts of scattering amplitude. This important problem will be investigated in a forthcoming paper.

Acknowledgments. The authors greatly acknowledge enlightening discussions with E. Balslev, J. V. Corbett, A. Grossman and B. Simon.

\section{Appendix I}

The family of resolvents

$$
\left(e^{-2 \theta} H_{0}+V(\theta)-z\right)^{-1}=e^{2 \theta} R_{0}\left(e^{2 \theta} z\right)\left[1+e^{2 \theta} V(\theta) R_{0}\left(e^{2 \theta} z\right)\right]^{-1}
$$

is analytic in $\theta \in \mathcal{O}^{\varepsilon}$ and meromorphic in $z \in \mathbb{C}_{i \varepsilon}^{++}$where $\varepsilon$ is arbitrarily fixed, $0<\varepsilon<\Pi / 4$. We investigate real poles in $z$ and residues for such poles. These poles are multivalent analytic functions of $\theta$ in $\mathcal{O}^{+\varepsilon}$ (in fact analytic functions of $\theta^{1 / p}$ for some integer $\left.p[10]\right)$; furthermore they are fixed when $\theta$ varies on lines parallel to $R$, for such variations preserve the spectrum of $e^{2 \theta} V(\theta) R_{0}\left(e^{2 \theta} z\right)$ (unitary equivalence). Accordingly the location of poles in $\mathbb{C}_{i \varepsilon}^{++}$do not depend on $\theta$. For a real pole $E$, the residue is given by

$$
P^{+}(E, \theta)=-\frac{1}{2 i \Pi} \int_{\Gamma_{E}} e^{2 \theta} R_{0}\left(e^{2 \theta} z\right)\left[1+e^{2 \theta} V(\theta) R_{0}\left(e^{2 \theta} z\right)\right]^{-1} d z
$$

where $\Gamma_{E}$ is a contour in $\mathbb{C}_{i \varepsilon}^{++}$enclosing $E$ (and no other pole) since $P^{+}(E, \theta)$ is the residue of a resolvent it is a projection operator. Using $\int_{\Gamma_{E}} R_{0}\left(e^{2 \theta} z\right) d z=0$ it can be rewritten as

$P^{+}(E, \theta)=\frac{1}{2 i \Pi} \int_{\Gamma_{E}} e^{4 \theta} R_{0}\left(e^{2 \theta} z\right) V(\theta) R_{0}\left(e^{2 \theta} z\right)\left[1+e^{2 \theta} V(\theta) R_{0}\left(e^{2 \theta} z\right)\right]^{-1} d z$

which implies that $P^{+}(E, \theta)$ is compact. Finally the integrand being analytic in $\theta$, the family $P^{+}(E, \theta)$ is analytic in $\mathcal{O}^{+\varepsilon}$. Summing up and since 
$\varepsilon$ was arbitrary, the family $P^{+}(E, \theta)$ is an analytic family of compact projection operators in $\mathcal{O}^{+}$. In the same way one can define a family $P^{-}(E, \theta), \theta \in \mathcal{O}^{-}$, having similar properties.

\section{Appendix II}

$\forall \Phi \in \mathscr{D}$ and $\theta \in S_{a}$ we have

$$
\|\Phi(\theta)\|^{2} \leqq\|\Phi(-i a)\|^{2}+\|\Phi(i a)\|^{2}
$$

Proof. Let us consider the spectral decomposition of the unitary operator

$$
U(\theta)=\int_{-\infty}^{+\infty} e^{i \lambda \theta} d E(\lambda)
$$

$\forall \Phi \in \mathscr{D}$ and $\theta \in S_{a}$ one has

$$
\|\Phi(\theta)\|^{2}=\int_{-\infty}^{+\infty} e^{-2 \lambda \operatorname{Im} \theta} d\|E(\lambda) \Phi\|^{2} .
$$

Let us consider $\operatorname{Im} \theta \geqq 0$ then

and

$$
\int_{-\infty}^{0} e^{-2 \lambda \operatorname{Im} \theta} d\|E(\lambda) \Phi\|^{2} \leqq \int_{-\infty}^{0} e^{-2 \lambda \theta} d\|E(\lambda) \Phi\|^{2} \leqq\|\Phi(i a)\|^{2}
$$

$$
\int_{0}^{\infty} e^{-2 \lambda \operatorname{Im} \theta} d\|E(\lambda) \Phi\|^{2} \leqq \int_{0}^{\infty} e^{2 \lambda \theta} d\|E(\lambda) \Phi\|^{2} \leqq\|\Phi(-i a)\|^{2} .
$$

Then we have

$$
\|\Phi(\theta)\|^{2} \leqq\|\Phi(-i a)\|^{2}+\|\Phi(i a)\|^{2} .
$$

The same result holds for $\operatorname{Im} \theta \leqq 0$.

We conclude that $\forall \Phi \in \mathscr{D}$ and $\theta \in S_{a}$

$$
\|\Phi(\theta)\|^{2} \leqq\|\Phi(-i a)\|^{2}+\|\Phi(i a)\|^{2} .
$$

\section{References}

1. Amrein, W., Misra, Ph., Martin. B.: On the asymptotic condition of scattering theory. Helv. Phys. Acta 43, 313 (1970).

2. Combes, J.M.: An algebraic approach to quantum scattering theory. Preprint Marseille.

3. Lavine, R. B.: Scattering theory for long range potentials. J. Functional Analysis 5, 368 (1970).

4. - Commutators and scattering theory, Part I: Repulsive interactions. Preprint Cornell University.

5. - Commutators and scattering theory, Part II: Class of one-body problem. Preprint Cornell University. 
6. Weidmann, J.: The virial theorem and its applications to the spectral theory of Schrödinger operators. Bull. Amer. Math. Soc. 73, 452 (1967).

7. Lovelace, C.: Three particle systems and unstable particles. Scottish Universities Summer School, Moorhouse (1963).

8. Bottino, Longoni, A., Regge, T.: Potential scattering for complex energy and angular momentum. Nuovo Cimento 23, 354 (1962).

9. Brown, L., Fivel, D., Lee, B., Sawyer, R.: Fredholm method in potential scattering and its application to complex angular momentum. Ann. Phys. 23, 187 (1963).

10. Steinberg, S.: Meromorphic families of compact operators. Arch. for Rat. Mechanics and analysis. 31, 5, 372 (1968).

11. Dunford-Schwartz: Linear operators I. New York: Interscience Publ. 1959.

12. Nelson, E.: Analytic vectors. Ann. Math. 70, 3 (1959).

13. Combes, J.M.: Relatively compact interactions in many particle system. Appendix I. Commun. math. Phys. 12, 283 (1969).

14. Kato: Perturbation theory for linear operators. Berlin-Heidelberg-New York: Springer 1966.

15. Wong, J.: On a condition for completness. J. Math. Phys. 10, 1438 (1969).

\author{
J. Aguilar \\ J. M. Combes \\ Centre de Physique Théorique \\ C.N.R.S. \\ 31 Chemin J. Aiguier \\ F-13 Marseille, France
}

